Research

\title{
Generalized anxiety disorder among Bangladeshi university students during COVID-19 pandemic: gender specific findings from a cross-sectional study
}

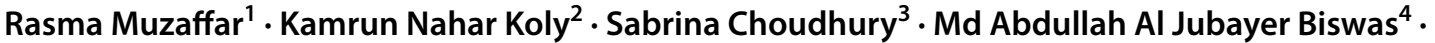 \\ Shirmin Bintay Kader ${ }^{5}$. Rehnuma Abdullah ${ }^{1}$. Umme Kawser ${ }^{6}$. M. Tasdik Hasan ${ }^{7,8,9}$. Darryn Williams ${ }^{10}$. \\ Ariful Bari Chowdhury ${ }^{1} \cdot$ Helal Uddin Ahmed ${ }^{11}$
}

Received: 29 October 2021 / Accepted: 24 January 2022

Published online: 08 February 2022

(c) The Author(s) 2022 OPEN

\begin{abstract}
In the current COVID-19 pandemic there are reports of deteriorating psychological conditions among university students in lower-middle-income countries (LMICs), but very little is known about the gender differences in the mental health conditions on this population. This study aims to assess generalized anxiety disorder (GAD) among university students using a gender lens during the COVID-19 pandemic. A cross-sectional study was conducted using web-based Google forms between May 2020 and August 2020 among 605 current students of two universities in Bangladesh. Within the total 605 study participants, 59.5\% (360) were female. The prevalence of mild to severe anxiety disorder was $61.8 \%$ among females and $38.2 \%$ among males. In the multivariable logistic regression analysis, females were 2.21 times more likely to have anxiety compared to males [AOR: 2.21; Cl 95\% (1.28-53.70); p-value: 0.004] and participants' age was negatively associated with increased levels of anxiety $(\mathrm{AOR}=0.17 ; 95 \% \mathrm{Cl}=0.05-0.57 ; p=0.001)$. In addition, participants who were worried about academic delays were more anxious than those who were not worried about it (AOR: $2.82 ; 95 \% \mathrm{Cl}$ $1.50-5.31, p=0.001)$. These findings of this study will add value to the existing limited evidence and strongly advocate in designing gender-specific, low-intensity interventions to ensure comprehensive mental health services for the young adult population of Bangladesh.
\end{abstract}

Keywords Mental health \& well-being $\cdot$ COVID-19 $\cdot$ Anxiety $\cdot$ Gender $\cdot$ Students

\section{Introduction}

The coronavirus disease (COVID-19) is a global public health emergency that has been detrimental to the overall population's mental health [1]. The preventive measures for COVID-19 transmission have had a direct negative bearing on global mental health of the world population, of which one quarter are youths aged 10-24 years [2,3]. Thus, it is unsurprising

Kamrun Nahar Koly, koly@icddrb.org; Shirmin Bintay Kader, sharmin.rim@gmail.com | ${ }^{1}$ Department of Public Health, School of Health \& Life Sciences, North South University, Dhaka, Bangladesh. ${ }^{2}$ International Centre for Diarrhoeal Disease Research, Bangladesh (icddr,b), Dhaka, Bangladesh. ${ }^{3}$ National Institute of Preventive and Social Medicine (NIPSOM), Dhaka, Bangladesh. ${ }^{4}$ Department of Statistics, University of Dhaka, Dhaka, Bangladesh. ${ }^{5}$ University of Saskatchewan, Saskatoon, Canada. ${ }^{6}$ Department of Educational and Counselling Psychology, University of Dhaka, Dhaka, Bangladesh. ${ }^{7}$ Department of Public Health, State University of Bangladesh, Dhaka, Bangladesh. ${ }^{8}$ Jeeon Bangladesh Ltd., Dhaka, Bangladesh. ${ }^{9}$ Department of Primary Care and Mental Health, University of Liverpool, Liverpool, UK. ${ }^{10}$ London School of Hygiene and Tropical Medicine, London, UK. ${ }^{11}$ National Institute of Mental Health, Bangladesh, Dhaka, Bangladesh. 
that in a lower-middle-income country like Bangladesh, COVID-19 has also affected the mental health of young adults group [4]. Evidence suggests that gender differences exist in common mental health conditions among varying age groups, in particular, generalized anxiety disorder (GAD) and depression are more prevalent among females [5]. Although gender has been shown to be an important social determinant, due consideration of this factor has been missing from the mental health burden investigations, particularly in this COVID-19 pandemic.

University students, already contending with the mental strain of tertiary educational curriculum, have faced myriad additional disruptions due to measures to curb the pandemic, such as strict lockdowns [6]. Disruptions in social support connections, usual daily routines, academic delay, and fear of COVID-19 infection have further deteriorated students' mental health status [7]. Gender has showed a statistically significant association with mental health disorders, alongside the other major social determinants such as age, education, marital status, income level, occupation etc. [8]. Studies during the COVID-19 pandemic showed that in high-income countries, young female adults attending universities were more susceptible to common mental health conditions such as anxiety, depressive symptoms, and post-traumatic stress disorder (PTSD) [8-12]. Similarly, South Asian countries like Pakistan and India also reported a higher number of young female university students aged 18-25 years compared to men, suffering from these psychological conditions due to COVID-19 [11, 13].

Furthermore, in Bangladesh, educational institution's pandemic-related restrictions such as lockdown have left 3.15 million university students uncertain about their future [14-16]. Students are always comfortable in interaction with their peers, teachers and enjoy the university campus atmosphere, but the lockdown has created a massive void for these students with consequent home-based, online and often sedentary lifestyles [7].

Existing literatures that investigated mental health conditions of the students during the pandemic were either conducted among the medical students, or included students from universities on the peripheries of Bangladesh. None discussed gender differences in terms of mental health disorders investigated [17-19].

Hence, this study aims to assess generalized anxiety disorder among university students during the COVID-19 pandemic using a gender lens. The findings can potentially foster the design of appropriate gender-based mental health interventions well-suited to educational institutions tailored to the special need of both men and women.

\section{Materials and methods}

\subsection{Study design}

A cross-sectional online survey was implemented among the students of two prominent public and private universities in Bangladesh (North South University and University of Dhaka) between May and August 2020. The research team included two of the faculties from both universities who played an instrumental role in developing a list of respondents. The list comprised email addresses and Facebook network accounts of potential respondents. The research team dispatched a web-based survey invitation to the students from list. In addition to this, the survey was also posted in the university associated Facebook groups as well as other smaller groups tied to the selected universities. The invitation contained a brief background, objectives, rights of the participants and the declaration of maintaining confidentiality. A contact address was also shared in the form, if any student had any queries before, during or after the survey. The students who accepted the invitation to participate in the survey browsed the invitation link and provided their responses.

\subsection{Sample size and data collection}

The sample size was calculated using a single population proportion formula by considering the proportion of moderate to severe anxiety levels as $61.0 \%$ among university students in Bangladesh measured by GAD-7 [20]. In addition, assuming a $95 \%$ confidence level, $5 \%$ absolute precision, $10 \%$ non-response rates, and a 1.49 -design effect, the estimated sample size is 605 [21]. Due to COVID-19 pandemic, government-imposed lockdown was going on during the data collection period. Consequently, our research team decided to conduct survey using online platform. The investigators were inclusive, open \& circulate the form periodically for a maximum reach. Email addresses of the interested participants were collected to check their studentship for the reliability of the data. The research team approached 2432 students, 
and a total of 811 responses were received. We finally analyzed data from 605 respondents, excluding the others due to inconsistency and missing information. The data collection flow chart is shown in Fig. 1.

\subsection{Research tool}

A self-reported, structured online questionnaire (both in Bangla and English) was distributed to the study participants along with an informed consent form. Each questionnaire had five dimensions: sociodemographic factors, questions on recreational activities, sleeping pattern, academic related information and the GAD-7 Scale. In the sociodemographic section, respondents were asked about age, gender, type of their residential areas, education level, marital status, occupation, number of family members, number of people within close circles infected by COVID-19, and feelings of distress due to the pandemic. Also, respondents were asked questions regarding their sleeping pattern and daily life activities such as sources of entertainment, feelings of concern evoked by reporting on the COVID-19 pandemic on television, levels of physical exercise, and engagement in household chores. Both English and Bangla versions of the seven-item generalized anxiety disorder (GAD-7) scale, validated in the Bangladeshi context, was used to identify the presence of anxiety among the participants $[17,22,23]$. Each item of the GAD-7 scale is scored on of a four-point Likert scale with; zero indicates no difficulty at all and three indicating extreme difficulty. Global summation of the scores results in a

Fig. 1 Data collection flow chart

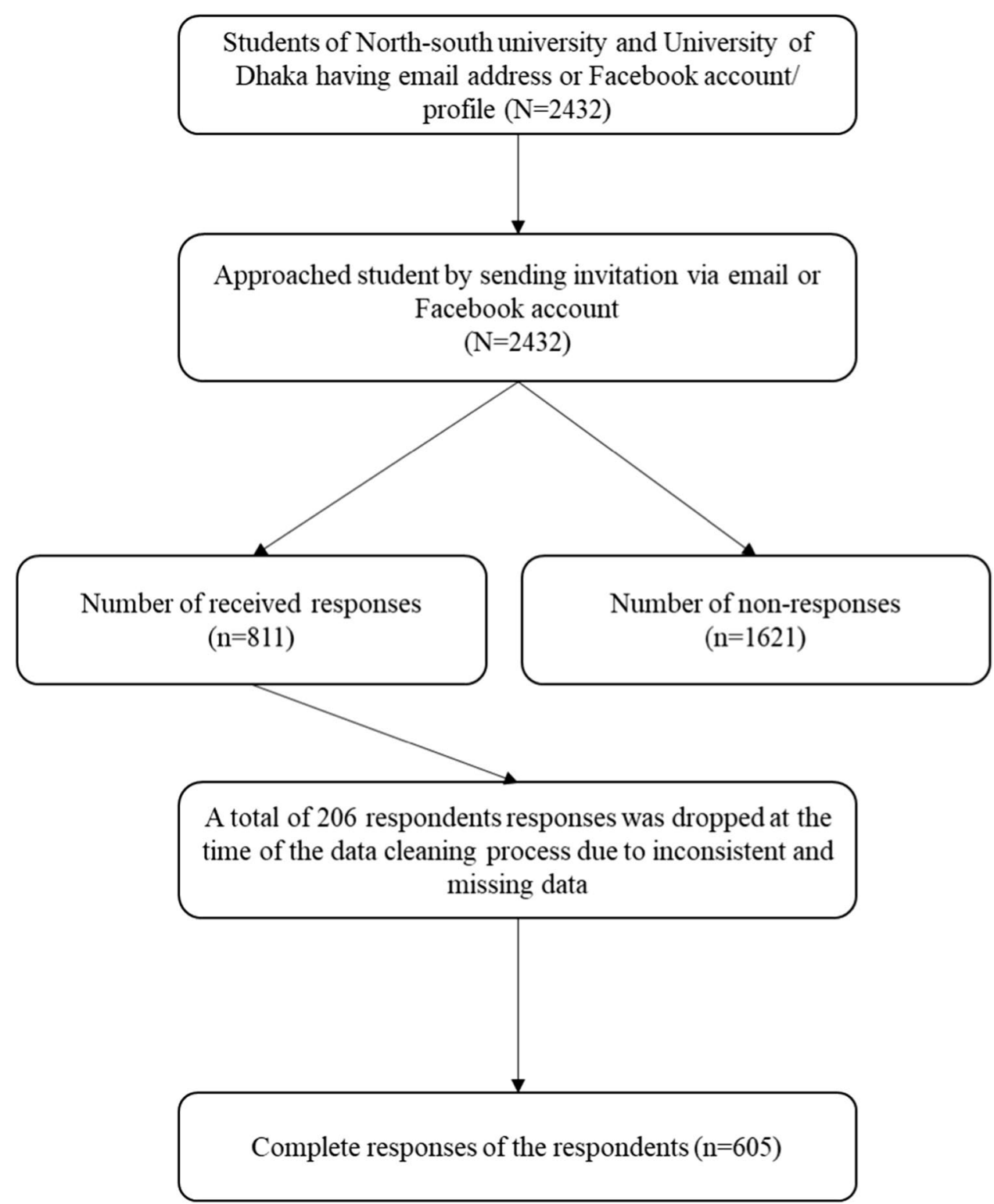


total between zero and 21, reflecting the severity of anxiety. A score of four or less indicated a minimal/no level of anxiety [24]. Scores between five and nine, inclusive, reflected mild anxiety levels, between 10 to 14, reflected moderate anxiety, and $>15$ indicated severe anxiety [22]. Anxiety scores were then binarized based on a threshold score of 4 [24]. Participants with a GAD score less than or equal to 4 were deemed to have experienced no anxiety whilst the rest were considered to have experienced mild to severe levels of anxiety [24]. The study was conducted in line with the Checklist for Reporting Results of Internet E-Surveys (CHERRIES) guideline [25].

\subsection{Statistical analysis}

The final dataset contained information of 605 students. For, further analysis of the data, we then used frequency and percentages for categorical variables, whilst, for continuous variables, mean and standard deviation (SD) were used to present descriptive statistics. We also conducted the chi-square test or fisher exact test (as appropriate) for categorical variables and Mann-Whitney test, Shapiro-Wilk test for the continuous variables to explore the significant gender-specific differences between gender-specific statistics and anxiety levels of the respondents. Multivariable logistic regressions were used to investigate gender-specific anxiety after controlling for socio-demographic factors, recreational activities, sleeping pattern, academic status [26]. In bivariate analysis, factors or variables with a p-value less than 0.05 were included in the multivariable regression model. The crude odds ratio (COR) for bivariable logistic models was reported with a 95\% confidence interval, while the adjusted odds ratio (AOR) for multivariable logistic regression was reported with a 95\% confidence interval. The Hosmer and Lemeshow goodness of fit tests were used to determine how well our model predicted the data. The Pseudo R-squares, such as the Cox and Snell's R-Square, the Nagelkerke's R-Square, and the McFadden's R-Square were utilized to indicate the percentage of variance in the outcome variable explained by the model. P-values less than 0.05 to indicate the statistical significance of the association. The analysis was performed using the SPSS software (version 25.0).

\section{Results}

\subsection{Characteristics of the sample}

Among the 605 study participants, $59.5 \%$ were female, where $40.5 \%$ were male. The mean age of the students was 23.1 years ( $S D \pm 3.4$ ). Most of them (96.4\%) lived with their families during the COVID-19 pandemic, $90.9 \%$ were unmarried and $86.3 \%$ of students lived in urban areas (Table 1). The percentage of sleeping pattern that affected the daily life activities are similar among male (40\%) and female (42\%). A higher percentage of female students felt worried about academic delays than the males (65.3\% vs $54.7 \%)$. The usage of Facebook as social media platform was higher among males (81.2\%) than female users (71.4\%) (Table 1).

\subsection{Prevalence of gender-specific anxiety disorder}

The overall mean of GAD-7 score of the participants was $9.6(\mathrm{SD}=4.6)$; among women it was $10.3(\mathrm{SD}=4.8)$; whereas it was 8.4 ( $(\mathrm{SD}=4.2)$ for men. The distribution of women across the different severities of anxiety was as follows: minimum (9.2\%), mild (39.7\%), moderate (30.3\%) and severe (20.8\%). On the other hand, about $17.5 \%, 45.3 \%, 28.6 \%$, and $8.6 \%$ of male students were suffering from minimum, mild, moderate and severe anxiety respectively (Fig. 2).

We found that 529 of 605 student participants reported a mild to severe degree of anxiety, with women accounting for $61.8 \%(n=327)$ and men accounting for $38.2 \%(n=202)$ (Table 1). The prevalence of anxiety among women participants who were from private university, unmarried, staying with family and in urban areas were respectively $57.8 \%, 87.8 \%$, $98.2 \%$ and $91.1 \%$, and for men, these were $43.1 \%, 97.0 \%, 93.6 \%$ and $77.7 \%$ respectively. The prevalence of anxiety was $45.6 \%$ and $67.3 \%$, among the women who reported an interruption in daily life activities due to sleeping patterns and worry about academic delays. Whereas for the men, the prevalence was $42.1 \%$ and $59.4 \%$, respectively (Table 2). 
Table 1 General Demographic information about the study participants, 2020 Dhaka Bangladesh

\begin{tabular}{|c|c|c|c|}
\hline Variables & Female, n (\%) & Male, n (\%) & Total, n (\%) \\
\hline Total & $360(59.5)$ & $245(40.5)$ & $605(100)$ \\
\hline \multicolumn{4}{|l|}{ Age in year } \\
\hline Mean \pm SD & $23.3 \pm 3.8$ & $22.8 \pm 2.8$ & $23.1 \pm 3.4$ \\
\hline$\leq 20$ & $67(18.61)$ & 47 (19.18) & $114(18.84)$ \\
\hline $21-26$ & $254(70.56)$ & $179(73.06)$ & $433(71.57)$ \\
\hline$\geq 27$ & $39(10.83)$ & $19(7.76)$ & $58(9.59)$ \\
\hline \multicolumn{4}{|l|}{ Mode of living } \\
\hline Alone/ Hostel & $6(1.7)$ & $16(6.5)$ & $22(3.6)$ \\
\hline Family & $354(98.3)$ & $229(93.5)$ & $583(96.4)$ \\
\hline \multicolumn{4}{|l|}{ Type of residential area } \\
\hline Rural & $31(8.6)$ & $52(21.2)$ & $83(13.7)$ \\
\hline Urban & $329(91.4)$ & $193(78.8)$ & $522(86.3)$ \\
\hline \multicolumn{4}{|l|}{ Educational institution } \\
\hline Public university & $155(43.1)$ & $135(55.1)$ & 290 (47.9) \\
\hline Private university & 205 (56.9) & $110(44.9)$ & $315(52.1)$ \\
\hline \multicolumn{4}{|l|}{ Education level } \\
\hline Undergraduate & $252(70)$ & $179(73.1)$ & 431 (71.2) \\
\hline Postgraduate & $108(30)$ & $66(26.9)$ & $174(28.8)$ \\
\hline \multicolumn{4}{|l|}{ Year of study (only for undergraduate) } \\
\hline 1st year & $30(11.9)$ & $26(14.5)$ & $56(13)$ \\
\hline 2nd year & $60(23.8)$ & $43(24)$ & $103(23.9)$ \\
\hline 3rd year & $60(23.8)$ & $50(27.9)$ & $110(25.5)$ \\
\hline Final year & $102(40.5)$ & $60(33.5)$ & $162(37.6)$ \\
\hline \multicolumn{4}{|l|}{ Marital status } \\
\hline Unmarried & $316(87.8)$ & $234(95.5)$ & $550(90.9)$ \\
\hline Married & $44(12.2)$ & $11(4.5)$ & $55(9.1)$ \\
\hline \multicolumn{4}{|l|}{ Current occupation } \\
\hline Unemployed & $38(10.6)$ & $31(12.7)$ & $69(11.4)$ \\
\hline Solely Student & $252(70)$ & $168(68.6)$ & $420(69.4)$ \\
\hline Service & $46(12.8)$ & $32(13.1)$ & 78 (12.9) \\
\hline Business/Self-employed & $24(6.7)$ & $14(5.7)$ & $38(6.3)$ \\
\hline \multicolumn{4}{|l|}{ Number of members in the family } \\
\hline$\leq 3$ & $65(18.10)$ & $41(16.7)$ & $106(17.5)$ \\
\hline $4-5$ & $227(63.00)$ & $164(66.9)$ & $391(64.6)$ \\
\hline$\geq 6$ & $68(18.90)$ & $40(16.4)$ & $108(17.9)$ \\
\hline \multicolumn{4}{|l|}{ Close circle exposed by COVID-19 } \\
\hline Yes & $223(61.90)$ & $131(53.5)$ & $354(58.5)$ \\
\hline No & $122(33.90)$ & $101(41.2)$ & $223(36.9)$ \\
\hline No idea & $15(4.20)$ & $13(5.3)$ & $28(4.6)$ \\
\hline \multicolumn{4}{|c|}{ Himself /herself infected with COVID-19 } \\
\hline Yes & $23(6.4)$ & $9(3.7)$ & $32(5.3)$ \\
\hline No & $337(93.6)$ & $236(96.3)$ & $573(94.7)$ \\
\hline \multicolumn{4}{|l|}{ Worried about the pandemic situation } \\
\hline Yes & $190(52.8)$ & $125(51)$ & $315(52.1)$ \\
\hline Sometimes & $156(43.3)$ & $103(42)$ & $259(42.8)$ \\
\hline No & $14(3.9)$ & $17(7)$ & $31(5.1)$ \\
\hline \multicolumn{4}{|l|}{ Way to keep on with social networking } \\
\hline Facebook & $257(71.4)$ & 199 (81.2) & $456(75.4)$ \\
\hline I don't feel like to contact anyone & $41(11.4)$ & $15(6.1)$ & $56(9.3)$ \\
\hline Phone calls (either video/audio) & $62(17.2)$ & 31 (12.7) & 93 (15.4) \\
\hline
\end{tabular}


Table 1 (continued)

\begin{tabular}{|c|c|c|c|}
\hline Variables & Female, n (\%) & Male, $\mathrm{n}(\%)$ & Total, n (\%) \\
\hline \multicolumn{4}{|c|}{ Feeling worried about COVID-19 news on television } \\
\hline Yes & $168(46.7)$ & $122(49.8)$ & $290(47.9)$ \\
\hline No & $192(53.3)$ & $123(50.2)$ & $315(52.1)$ \\
\hline \multicolumn{4}{|c|}{ Maintaining a regular physical exercise } \\
\hline Yes & $57(15.8)$ & $54(22)$ & $111(18.3)$ \\
\hline Occasionally & $170(47.2)$ & $88(35.9)$ & $258(42.6)$ \\
\hline No & $133(36.9)$ & $103(42)$ & $236(39)$ \\
\hline \multicolumn{4}{|l|}{ Doing household chores } \\
\hline Yes & $209(58.1)$ & $100(40.8)$ & $309(51.1)$ \\
\hline Occasionally & $120(33.3)$ & $96(39.2)$ & $216(35.7)$ \\
\hline No & $31(8.6)$ & $49(20)$ & $80(13.2)$ \\
\hline \multicolumn{4}{|c|}{ Feeling any difficulty in sleeping at night } \\
\hline Severe & $127(35.3)$ & $67(27.4)$ & $194(32.1)$ \\
\hline Moderate & $94(26.1)$ & $75(30.6)$ & $169(27.9)$ \\
\hline Mild & $139(38.6)$ & $103(42)$ & $242(40)$ \\
\hline \multicolumn{4}{|c|}{ Stressed about the sleeping pattern } \\
\hline Always & $97(26.9)$ & $59(24.1)$ & $156(25.8)$ \\
\hline Often & $52(14.4)$ & $33(13.5)$ & $85(14)$ \\
\hline Sometime & $144(40)$ & $92(37.6)$ & $236(39)$ \\
\hline Not at all & $67(18.6)$ & $61(24.9)$ & $128(21.2)$ \\
\hline \multicolumn{4}{|c|}{ Current sleeping pattern affecting daily life } \\
\hline Always & $150(41.7)$ & $98(40)$ & $248(41)$ \\
\hline Often & $9(2.5)$ & $10(4.1)$ & $19(3.1)$ \\
\hline Sometime & $148(41.1)$ & $88(35.9)$ & $236(39)$ \\
\hline Not at all & $53(14.7)$ & $49(20)$ & $102(16.9)$ \\
\hline \multicolumn{4}{|l|}{ Worried about academic delays } \\
\hline Yes & $235(65.3)$ & $134(54.7)$ & $369(61)$ \\
\hline Sometimes & $82(22.8)$ & $53(21.6)$ & $135(22.3)$ \\
\hline No & $43(11.9)$ & $58(23.7)$ & $101(16.7)$ \\
\hline \multicolumn{4}{|c|}{ Have any pre-existing psychological issues } \\
\hline Yes & $82(22.8)$ & $46(18.8)$ & $128(21.2)$ \\
\hline I feel like but not sure about it & $124(34.4)$ & $70(28.6)$ & $194(32.1)$ \\
\hline No & $154(42.8)$ & $129(52.7)$ & $283(46.8)$ \\
\hline \multicolumn{4}{|c|}{ Proportion of mild to severe level of anxiety } \\
\hline Yes & $327(90.80)$ & $202(82.40)$ & $529(87.40)$ \\
\hline No & $33(9.20)$ & $43(17.50)$ & $76(12.60)$ \\
\hline
\end{tabular}

\subsection{Factors associated with gender difference in the prevalence of anxiety}

Table 3 represents the odds ratios from the logistic regression analysis. The multivariable logistic regression model included age in years, sex, type of residential area, mode of living, educational institution, and marital status as covariates. These covariates were found to be statistically significant in a bivariable analysis. We found in the adjusted model that age in years, sex and worry about academic delays were significantly associated with mild to severe levels of anxiety (Table 3). Female university students were 2.21 times more likely [AOR: 2.21 ; Cl 95\% (1.28-53.70); p-value: 0.004 ] to report anxiety than male students. Likewise, participants who reported worrying about their academic delays had about 2.82 times greater odds of anxiety compared to the counter reference category. Other than that, lower probabilities of anxiety were found among students aged 21-26 years and more than or equal to 27 . 
Fig. 2 Percentage distribution of severity level of anxiety among students

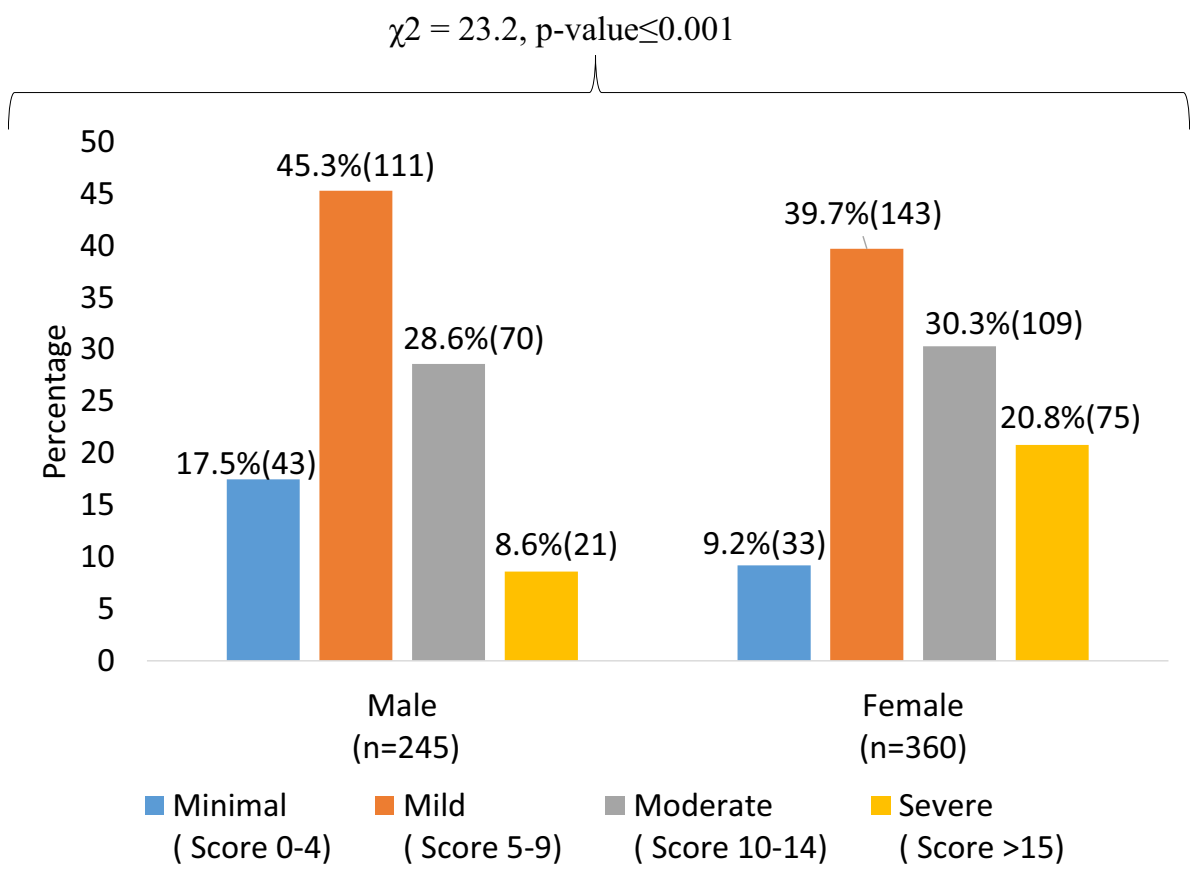

\section{Discussion}

Evidence shows that major pandemic or epidemic outbreaks have always been associated with a spike in mental health consequences which usually deteriorates further as the outbreaks progress [17, 18, 27-30]. Similarly, countries worldwide have been affected much with multiple waves of the COVID-19 pandemic and each wave has depicted different trajectories of daily new COVID-19 cases, transmission and mortality rates [31]. To date, Bangladesh has experienced two waves of the pandemic with the second wave being more devastating [32]. Aligning with this, prior studies have reported of declining mental health conditions during the second wave of COVID-19 compared to the first wave of COVID-19 [29,33]. However, it also has to be considered that the first wave of COVID-19 did create immense tension among the population due to sudden outbreak of a new disease leading to strict lockdown, uncertainties of the infection, confinement of social spaces and management of professional duties [34]. Hence, our study conducted was during the first wave of the pandemic to assess the mental health conditions among the university students in Bangladesh during this period.

Furthermore, in prior studies, gender disparities also existed among the prevalence of mental health conditions. Similarly in Bangladesh, gender differences were significantly associated with increased anxiety especially among women and students during both the waves of pandemic [27,35]. Additionally in a prior study, gender and its dependence on cross-cultural differences has essentially influenced the prevalence of mental health conditions during this pandemic [36]. With very limited number of studies using a gender specific lens. This is one of the very few studies that has examined student mental health from urban areas involving two of the largest universities of Bangladesh through a gender-specific lens. By analyzing anxiety disorders among students during the first wave of pandemic through a gender-specific lens, this study will support in devising comprehensive interventions in case of future emergencies.

From the pre-COVID-19 period, university students from Bangladesh were reported to have higher levels of common mental health issues than other population groups [17, 19, 23]. Furthermore, in comparison to pre-COVID-19 era, possible factors such as fear of infection, fabricated news, and academic delay, social and financial insecurities led to a rise in mental health conditions among university students during the pandemic $[6,16,17,37-41]$. Likewise, our study also revealed a higher over-all prevalence of mild to severe generalized anxiety disorder among women $61.8 \%(N=327)$ relative to men $38.2 \%(N=202)$ within the university students population during this pandemic.

Concurrent evidence from LMICs has affirmed that the prescribed gender roles have resulted in young females being burdened with domestic duties, financial dependency, lesser coping strategies, and more uncertainty during the lockdown, making them prone to developing mental health symptoms [18, 30, 42-45]. A plethora of studies from worldwide 
Table 2 Prevalence of mild to severe levels of anxiety risks measured by GAD-7 among the male and female students, during COVID-19 pandemic according to their characteristics, 2020 Dhaka Bangladesh

\begin{tabular}{|c|c|c|c|c|c|}
\hline \multirow[t]{3}{*}{ Variables } & \multicolumn{2}{|c|}{$\begin{array}{l}\text { Without anxiety (minimal/normal) } \\
(\mathrm{N}=76)\end{array}$} & \multicolumn{3}{|c|}{$\begin{array}{l}\text { With anxiety (mild to severe) } \\
(\mathrm{N}=529)\end{array}$} \\
\hline & Female $(\mathrm{N}=33)$ & Male $(\mathrm{N}=43)$ & Female $(\mathrm{N}=327)$ & Male $(\mathrm{N}=202)$ & $p$-value \\
\hline & n (\%) & n (\%) & n (\%) & n (\%) & \\
\hline \multicolumn{6}{|l|}{ Age in year } \\
\hline Mean \pm SD & $25.03 \pm 3.90$ & $23.86 \pm 3.68$ & $23.15 \pm 3.79$ & $22.62 \pm 2.54$ & \\
\hline$\leq 20$ & $0(0.00)$ & $6(13.95)$ & $67(20.49)$ & $41(20.30)$ & 0.580 \\
\hline $21-26$ & $25(75.76)$ & $32(74.42)$ & $229(70.03)$ & $147(72.77)$ & \\
\hline$\geq 27$ & $8(24.24)$ & $5(11.63)$ & $31(9.48)$ & $14(6.93)$ & \\
\hline \multicolumn{6}{|l|}{ Mode of living } \\
\hline Alone/Hostel & $0(0)$ & $3(6.98)$ & $6(1.83)$ & $13(6.44)$ & 0.006 \\
\hline Family & $33(100.0)$ & $40(93.02)$ & $321(98.17)$ & $189(93.56)$ & \\
\hline \multicolumn{6}{|l|}{ Type of residential area } \\
\hline Rural & $2(6.06)$ & $7(16.28)$ & $29(8.87)$ & $45(22.28)$ & $<0.001$ \\
\hline Urban & 31 (93.94) & $36(83.72)$ & $298(91.13)$ & $157(77.72)$ & \\
\hline \multicolumn{6}{|l|}{ Educational institution } \\
\hline Public university & $17(51.52)$ & $20(46.51)$ & $138(42.20)$ & $115(56.93)$ & 0.001 \\
\hline Private university & $16(48.48)$ & $23(53.49)$ & $189(57.80)$ & $87(43.07)$ & \\
\hline \multicolumn{6}{|l|}{ Education level } \\
\hline Undergraduate & $17(51.52)$ & $26(60.47)$ & $235(71.87)$ & $153(75.74)$ & 0.327 \\
\hline Postgraduate & $16(48.48)$ & $17(39.53)$ & $92(28.13)$ & $49(24.26)$ & \\
\hline \multicolumn{6}{|c|}{ Year of study (only for undergraduate) } \\
\hline 1st year & $1(5.88)$ & $4(15.38)$ & $29(12.34)$ & $22(14.38)$ & 0.516 \\
\hline 2nd year & $1(5.88)$ & $6(23.08)$ & $59(25.11)$ & $37(24.18)$ & \\
\hline 3rd year & $3(17.65)$ & $5(19.23)$ & $57(24.26)$ & $45(29.41)$ & \\
\hline Final year & $12(70.59)$ & $11(42.31)$ & $90(28.30)$ & 49 (32.03) & \\
\hline \multicolumn{6}{|l|}{ Marital status } \\
\hline Unmarried & $29(87.88)$ & $38(88.37)$ & $287(87.77)$ & $196(97.03)$ & $<0.001$ \\
\hline Married & $4(12.12)$ & $5(11.63)$ & $40(12.23)$ & $6(2.97)$ & \\
\hline \multicolumn{6}{|l|}{ Current occupation } \\
\hline Unemployed & $4(12.12)$ & $8(18.60)$ & $34(10.40)$ & $23(11.39)$ & 0.889 \\
\hline Solely Student & $22(66.67)$ & $24(55.81)$ & $230(70.34)$ & $144(71.29)$ & \\
\hline Service & $6(18.18)$ & $8(18.60)$ & $40(12.23)$ & $24(11.88)$ & \\
\hline Business/Self-employed & $1(3.03)$ & $3(6.98)$ & $23(7.03)$ & $11(5.45)$ & \\
\hline \multicolumn{6}{|l|}{ Number of family members } \\
\hline$\leq 3$ & $6(18.18)$ & $7(16.28)$ & $59(18.04)$ & $34(16.83)$ & 0.223 \\
\hline $4-5$ & $20(60.61)$ & $23(53.49)$ & $207(60.30)$ & $141(69.80)$ & \\
\hline$\geq 6$ & $7(21.21)$ & $13(30.23)$ & $61(18.65)$ & $27(13.37)$ & \\
\hline \multicolumn{6}{|c|}{ Close circle exposed by COVID-19 } \\
\hline No & $12(36.36)$ & $23(53.49)$ & $110(33.64)$ & $78(38.61)$ & 0.365 \\
\hline Yes & $20(60.61)$ & $18(41.86)$ & $203(62.08)$ & $113(55.94)$ & \\
\hline No idea & $1(3.03)$ & $2(4.65)$ & $14(4.28)$ & $11(5.45)$ & \\
\hline \multicolumn{6}{|c|}{ Himself /herself infected with COVID-19 } \\
\hline No & $33(100.0)$ & $41(95.35)$ & $304(92.97)$ & $195(96.53)$ & 0.085 \\
\hline Yes & $0(0)$ & $2(4.65)$ & $23(7.03)$ & $7(3.47)$ & \\
\hline \multicolumn{6}{|c|}{ Worried about the pandemic situation } \\
\hline No & $4(12.12)$ & $5(11.63)$ & $10(3.06)$ & $12(5.94)$ & 0.237 \\
\hline Sometimes & $20(60.61)$ & $26(60.47)$ & $136(41.59)$ & $77(38.12)$ & \\
\hline Yes & $9(27.27)$ & $12(27.91)$ & $181(55.35)$ & $113(55.94)$ & \\
\hline
\end{tabular}


Table 2 (continued)

\begin{tabular}{|c|c|c|c|c|c|}
\hline \multirow[t]{3}{*}{ Variables } & \multicolumn{2}{|c|}{$\begin{array}{l}\text { Without anxiety (minimal/normal) } \\
(\mathrm{N}=76)\end{array}$} & \multicolumn{3}{|c|}{$\begin{array}{l}\text { With anxiety (mild to severe) } \\
(\mathrm{N}=529)\end{array}$} \\
\hline & Female $(\mathrm{N}=33)$ & Male $(\mathrm{N}=43)$ & Female $(\mathrm{N}=327)$ & Male $(\mathrm{N}=202)$ & $p$-value \\
\hline & n (\%) & $\mathrm{n}(\%)$ & $\mathrm{n}(\%)$ & $\mathrm{n}(\%)$ & \\
\hline \multicolumn{6}{|l|}{ Way to keep on with social networking } \\
\hline Facebook & $23(69.70)$ & $32(74.42)$ & $234(71.56)$ & $167(82.67)$ & 0.010 \\
\hline I don't feel like to contact anyone & $3(9.09)$ & $4(9.30)$ & $38(11.62)$ & $11(5.45)$ & \\
\hline Phone calls (either video/audio) & $7(21.12)$ & $7(16.28)$ & $55(16.82)$ & $24(11.88)$ & \\
\hline \multicolumn{6}{|c|}{ Feeling worried about COVID-19 news on television } \\
\hline No & $22(66.67)$ & $30(69.77)$ & $170(51.99)$ & $93(46.04)$ & 0.184 \\
\hline Yes & $11(33.33)$ & $13(30.23)$ & $157(48.01)$ & 109 (53.96) & \\
\hline \multicolumn{6}{|c|}{ Maintaining a regular physical exercise } \\
\hline No & $8(24.24)$ & $16(37.21)$ & $125(38.23)$ & $87(43.07)$ & 0.078 \\
\hline Sometimes & $14(42.42)$ & $11(25.58)$ & $156(47.71)$ & $77(38.12)$ & \\
\hline Yes & $11(33.33)$ & $16(37.21)$ & $46(14.07)$ & $38(18.81)$ & \\
\hline \multicolumn{6}{|l|}{ Doing household chores } \\
\hline No & $3(9.09)$ & $8(18.60)$ & $28(8.56)$ & $41(20.30)$ & $<0.001$ \\
\hline Sometimes & $8(24.24)$ & $17(39.53)$ & $112(34.25)$ & $79(39.11)$ & \\
\hline Yes & $22(66.67)$ & $18(41.86)$ & $187(57.19)$ & $82(40.59)$ & \\
\hline \multicolumn{6}{|c|}{ Feeling any difficulty in sleeping at night } \\
\hline Severe & $2(6.06)$ & $9(20.93)$ & $125(38.23)$ & $58(28.71)$ & 0.082 \\
\hline Moderate & $6(18.18)$ & $12(27.91)$ & $88(26.91)$ & $63(31.19)$ & \\
\hline Mild & $25(75.76)$ & $22(51.16)$ & $114(34.86)$ & $81(40.10)$ & \\
\hline \multicolumn{6}{|l|}{ Stressed about the sleeping pattern } \\
\hline Always & $0(0.00)$ & $5(11.63)$ & $97(29.66)$ & $54(26.73)$ & 0.420 \\
\hline Often & $4(12.12)$ & $2(4.65)$ & $48(14.68)$ & $31(15.35)$ & \\
\hline Sometimes & $8(24.24)$ & $14(32.56)$ & $136(41.59)$ & $78(38.61)$ & \\
\hline Not at all & $21(63.64)$ & $22(51.16)$ & $46(14.07)$ & $39(19.31)$ & \\
\hline \multicolumn{6}{|c|}{ Current sleeping pattern affecting daily life } \\
\hline Always & $1(3.03)$ & $13(30.23)$ & $149(45.57)$ & $85(42.08)$ & 0.241 \\
\hline Often & $1(3.03)$ & $3(6.98)$ & $8(2.45)$ & $7(3.47)$ & \\
\hline Sometimes & $13(39.39)$ & $11(25.58)$ & $135(41.28)$ & 77 (37.12) & \\
\hline Not at all & $18(54.55)$ & $16(37.21)$ & $35(10.70)$ & $33(16.34)$ & \\
\hline \multicolumn{6}{|l|}{ Worried about academic delays } \\
\hline Yes & $15(45.45)$ & $14(32.56)$ & $220(67.28)$ & $120(59.41)$ & 0.001 \\
\hline Sometimes & $9(27.27)$ & $16(37.21)$ & $73(22.32)$ & $37(18.32)$ & \\
\hline No & $9(27.27)$ & $13(30.23)$ & $34(10.40)$ & $45(22.28)$ & \\
\hline \multicolumn{6}{|c|}{ Have any pre-existing psychological issues } \\
\hline No & $27(81.82)$ & $35(81.40)$ & $127(38.84)$ & $94(46.53)$ & 0.2 \\
\hline Yes & $1(3.03)$ & $5(11.63)$ & $81(24.77)$ & $41(20.30)$ & \\
\hline I feel like but not sure about it & $5(15.15)$ & $3(6.98)$ & $119(36.39)$ & $67(33.17)$ & \\
\hline
\end{tabular}

and Bangladesh, supported the idea that the prevalence of anxiety is higher among female students than males during the pandemic $[6,16-18,37-41,44,46-50]$. However, no statistical association between gender and psychological impact was explored and reported in several other studies from both Southern and Southeast Asian countries [20,51-53].

In multivariate analysis, our study reports that, age in years and being worried about academic delays were significantly associated with the level of anxiety. However, our study also revealed that anxiety and age are associated negatively among the women. Similar findings were reported in other LMICs where younger and newly enrolled students are already under the pressure of academic competition in a new environment, the lockdown has limited their scope of communication to build an inter-personal relationship with academic departments which maybe some 
Table 3 Multiple logistic regression analysis of the male and female students during COVID-19 pandemic with their characteristics, 2020 Dhaka Bangladesh

\begin{tabular}{|c|c|c|c|c|}
\hline \multirow[t]{2}{*}{ Variables } & \multicolumn{4}{|c|}{ Anxiety (mild to severe levels) } \\
\hline & $\operatorname{COR}(95 \% \mathrm{Cl})$ & $p$-value & AOR $(95 \% \mathrm{Cl})$ & $\mathrm{p}$-value \\
\hline \multicolumn{5}{|l|}{ Age in year } \\
\hline $21-26$ & $0.37(0.15-0.87)$ & 0.023 & $0.34(0.14-0.84)$ & 0.019 \\
\hline$\geq 27$ & $0.19(0.07-0.54)$ & 0.002 & $0.17(0.05-0.57)$ & 0.001 \\
\hline$\leq 20$ & Reference & & & \\
\hline \multicolumn{5}{|l|}{ Sex } \\
\hline Female & $2.11(1.29-3.43)$ & 0.003 & $2.21(1.28-3.7)$ & 0.004 \\
\hline Male & Reference & & Reference & \\
\hline \multicolumn{5}{|l|}{ Type of residential area } \\
\hline Urban & $1.21(0.58-2.53)$ & 0.611 & $0.68(0.31-1.50)$ & 0.343 \\
\hline Rural & Reference & & Reference & \\
\hline \multicolumn{5}{|l|}{ Mode of living } \\
\hline Family & $1.10(0.32-3.82)$ & 0.877 & $0.78(0.0 .20-3.02)$ & 0.722 \\
\hline Alone/Hostel & Reference & & Reference & \\
\hline \multicolumn{5}{|l|}{ Educational institution } \\
\hline Public university & $1.10(0.32-3.82)$ & 0.889 & $0.84(0.50-1.43)$ & 0.533 \\
\hline Private university & Reference & & Reference & \\
\hline \multicolumn{5}{|l|}{ Marital status } \\
\hline Married & $0.71(0.33-1.51)$ & 0.374 & $1.14(0.0 .42-3.1)$ & 0.797 \\
\hline Unmarried & Reference & & Reference & \\
\hline \multicolumn{5}{|l|}{ Way to keep on with social networking } \\
\hline Facebook & $1.29(0.69-2.44)$ & 0.429 & $1.34(0.68-2.64)$ & 0.39 \\
\hline I don't feel like to contact anyone & $1.24(0.47-3.29)$ & 0.665 & $1.03(0.37-2.87)$ & 0.955 \\
\hline Phone calls (either video/audio) & Reference & & Reference & \\
\hline \multicolumn{5}{|l|}{ Doing household chores } \\
\hline Yes & $0.93(0.46-1.91)$ & 0.849 & $0.89(0.41-1.94)$ & 0.773 \\
\hline Occasionally & $1.14(0.67-1.94)$ & 0.639 & $1.03(0.46-2.32)$ & 0.929 \\
\hline No & Reference & & Reference & \\
\hline \multicolumn{5}{|l|}{ Worried about academic delays } \\
\hline Yes & $3.27(1.78-5.98)$ & $<0.001$ & $2.82(1.50-5.31)$ & 0.001 \\
\hline Sometimes & $1.23(0.65-2.33)$ & 0.535 & $1.07(0.54-2.09)$ & 0.844 \\
\hline No & Reference & & & \\
\hline
\end{tabular}

COR: Crude odds ratio; AOR: Adjusted odds ratio; $\mathrm{Cl}$ : Confidence Interval Hosmer-Lemeshow tests: Chi-square ( $p$-value) = 7.11 (0.5246)

Cox and Snell's R-Square $=0.063$

Nagelkerke's R-Square $=0.119$

McFadden's R-Square $=0.086$

of the causes of anxiety at a younger age [54-56]. Whereas, some prior studies also reported of age being a positive factor for mental health conditions among students [54-57].

Due to lockdown related interruptions in routine teaching and assessments, the possibilities for the academic delay exist that can also jeopardise the expected graduation time of the students. In our study, participants who were worried about academic delays were significantly more likely to be anxious (AOR2.21 than who were not worried about it ( $p$-value: 0.004$)$. It has to be realized that the current pandemic has added uncertainties for the graduating students in finding jobs and enrolling in further education or may be finding internships [17]. Moreover, in South Asian countries like Bangladesh, women already have shorter time frame available for career development and marriage, the academic delay owing to the pandemic has further worsened scope thus leading to anxiety $[17,54,57,58]$. Studies both from global and South Asian countries also reported that students especially from the final years or the ones nearing graduation were found to be effected psychologically due to worrying about their academic curriculum [17, $58,59]$. As per prior studies, it can be concluded that women with education attainment are in socio-economically 
empowered position, which significantly reduces their risk of being mentally vulnerable [60-62]. Hence, it can be assumed that academic delays reduce the chances of women to be financially independent and empowered which in turn affects their mental health condition.

Also, in bivariate analysis factors like studying in private university, being unmarried, staying with the family, urban area residence, interrupted daily life activities due to sleeping pattern and worrying about academic delays contributed to higher prevalence of anxiety among females students. However, the findings were found to be non-significant while adjusting the confounding factors, which might be due to the limited sample size.

So, our study findings align with the fact that gender stereotypes have reinforced social stigma and constrained mental health help-seeking behavior in both men and women. Moreover, viewing mental health through the gender specific lens is crucial, especially for the female of developing countries. So, overlooking this bias among the youth could have drastic consequences on the national priority and productivity as the youth plays an important role in the country's economic growth. In addition, the study findings can be used to design need based and gender specific interventions for an effective mental health outcome among the youth population in Bangladesh.

\section{Strength and limitations}

Nevertheless, many limitations of the current study have been determined by the investigators. This was an online based survey therefore, there is no chance of observation and validation of the data given by the students. The limited sample size limits the generalizability of the findings. The identification of anxiety was entirely subjective; we did not apply the tools designed especially for the COVID-19 pandemic, for instance, the Coronavirus Anxiety Scale (CAS) [63]. The study also cannot establish the causal factors as it is a cross-sectional study. Self-reported measures may be responsible for recall and social desirability biases the present study does not provide any in-depth information behind the gender differences on the anxiety level among the students. Therefore, a qualitative analysis is highly recommended among the university students. In addition, there is also a possibility for selection bias associated with data collection method, as the survey could have attracted more students who either are suffering or free from anxiety disorder. Either way, this selection bias could have either inflated or underestimated the assessed prevalence.

However, despite all these limitations, the current study used the gender specific approach that was not used in any previously published studies focusing on mental health and COVID-19 among the students. The results showing that almost twice the number of female students were suffering from anxiety than male students suggesting that female student's mental health status should be monitored regularly. They might need holistic psychosocial interventions targeting women as a larger part of society, multi-disciplinary interventions involving primary care, judicial and legal fronts, and interventions. In addition, on comparing the impact of lockdown due to COVID-19 on the mental health status of students from private and public universities suggested that private university students might need additional psychosocial support during this pandemic. Thus, the current study viewed the student's mental health status via the gender lens, which was much needed to identify the vulnerable population group and design a gender-specific, social and policy level psychosocial interventions.

\section{Conclusion}

The COVID-19 pandemic has severe repercussion on mental health, especially among the young female university students in Bangladesh. Gender-specific and campus-based-low-intensity psychosocial interventions are needed to ensure effective mental health services for Bangladeshi university students. Besides, a nationwide longitudinal survey needs to be conducted to generate robust evidence that will sensitize the policymakers to formalize an urgent, feasible, and sustainable intervention model for an emergency condition such as a pandemic situation. Moreover, this study also creates the scope of comparative studies and doing further large-scale longitudinal studies assessing the mental health conditions considering the outbreak of new variants at different time points with a gender lens.

Acknowledgements The authors would like to express their gratitude towards the faculties of North South University, Dhaka, Bangladesh and University of Dhaka, Dhaka, Bangladesh and their university administered Facebook groups for spontaneous support with completion of data collection. They also want to express their gratitude to all the respondents who participated in this study voluntarily amid this COVID-19 situation. In addition, icddr, b is grateful to Bangladesh government, Canada, Sweden and the UK for providing core/unrestricted support. Lastly, 
the authors wish to express their sincere appreciation to all the research team members who took part in the research voluntarily without any incentive.

Authors' contributions The study was designed by KNK and KNK, RA, TH drafted the proposal according to the framework of North South University, Dhaka, Bangladesh. RA, SC, KNK, TH, UK, ABC coordinated data collection and JB, SBK, SC, DW cleaned, managed and analyzed the data. KNK drafted the first version of the manuscript and RA, RM assisted her throughout preparing the manuscript. KNK substantially refined the manuscript with assistance from RM, ABC, DW, UK, TH and HA critically supervised the team in all the stages. All authors read and approved the final manuscript.

Funding This work has received no funding.

Data availability The datasets generated and/or analyzed during the current study are available from the corresponding author on reasonable request.

Code availability Not applicable.

\section{Declarations}

Ethical approval and consent to participate Study participants were informed about a brief description of the study, including objectives, methods, declaration of maintaining confidentiality and rights to withdraw from study before starting the questionnaire electronically. Recruited participants were also informed that their participation in the study was voluntary and did not receive any compensation for their participation. This study was performed in line with the principles of the Declaration of Helsinki. Approval was granted by the Ethical Review Committee of Public Health Foundation, Bangladesh (PHF, BD) (01/2021).

Competing interests The authors declare no conflict of interest.

Open Access This article is licensed under a Creative Commons Attribution 4.0 International License, which permits use, sharing, adaptation, distribution and reproduction in any medium or format, as long as you give appropriate credit to the original author(s) and the source, provide a link to the Creative Commons licence, and indicate if changes were made. The images or other third party material in this article are included in the article's Creative Commons licence, unless indicated otherwise in a credit line to the material. If material is not included in the article's Creative Commons licence and your intended use is not permitted by statutory regulation or exceeds the permitted use, you will need to obtain permission directly from the copyright holder. To view a copy of this licence, visit http://creativecommons.org/licenses/by/4.0/.

\section{References}

1. Wang C, Pan R, Wan X, Tan Y, Xu L, Ho CS, et al. Immediate psychological responses and associated factors during the initial stage of the 2019 coronavirus disease (COVID-19) epidemic among the general population in China. Int J Environ Res Public Health. 2020;17(5):1729.

2. Chadda RK. Youth \& mental health: Challenges ahead. Indian J Med Res. 2018;148(4):359.

3. Ornell F, Schuch JB, Sordi AO, Kessler FH. "Pandemic fear" and COVID-19: mental health burden and strategies. Brazil J Psychiatry. 2020;42(3):232-5.

4. Banna MHA, Sayeed A, Kundu S, Christopher E, Hasan MT, Begum MR, et al. The impact of the COVID-19 pandemic on the mental health of the adult population in Bangladesh: a nationwide cross-sectional study. Int J Environ Health Res. 2020. https://doi.org/10.1080/09603 123.2020.1802409.

5. Minds Y. Coronavirus: Impact on young people with mental health needs. https://www.youngmindsorguk/media/3708/coronavirusreport_march2020pdf. 2020;21:2020.

6. Shama S, Ikbal J. Education during a pandemic: a feasibility study of online classes in Bangladesh to counteract potential study gaps caused by COVID-19 related lockdowns. SocArXiv. 2020.

7. Romero-Blanco C, Rodríguez-Almagro J, Onieva-Zafra MD, Parra-Fernández ML, Prado-Laguna MDC, Hernández-Martínez A, et al. Physical activity and sedentary lifestyle in university students: changes during confinement due to the Covid-19 pandemic. Int J Environ Res Public Health. 2020;17(18):6567.

8. Xiao H, Shu W, Li M, Li Z, Tao F, Wu X, et al. Social distancing among Medical students during the 2019 Coronavirus disease pandemic in China: disease awareness, anxiety disorder, depression, and behavioral activities. Int J Environ Res Public Health. 2020;17(14):5047.

9. Odriozola-González P, Planchuelo-Gómez Á, Irurtia MJ, de Luis-García R. Psychological effects of the COVID-19 outbreak and lockdown among students and workers of a Spanish university. Psychiatry Res. 2020;290:113108.

10. Patsali ME, Mousa D-PV, Papadopoulou EV, Papadopoulou KK, Kaparounaki CK, Diakogiannis I, et al. University students' changes in mental health status and determinants of behavior during the COVID-19 lockdown in Greece. Psychiatry Res. 2020;292:113298.

11. Sundarasen S, Chinna K, Kamaludin K, Nurunnabi M, Baloch GM, Khoshaim HB, et al. Psychological impact of COVID-19 and lockdown among university students in Malaysia: implications and policy recommendations. Int J Environ Res Public Health. 2020;17(17):6206.

12. Zhang $Y$, Zhang H, Ma X, Di Q. Mental health problems during the COVID-19 pandemics and the mitigation effects of exercise: a longitudinal study of college students in China. Int J Environ Res Public Health. 2020;17(10):3722.

13. Pandey U, Corbett G, Mohan S, Reagu S, Kumar S, Farrell T, et al. Anxiety, depression and behavioural changes in junior doctors and medical students associated with the coronavirus pandemic: a cross-sectional survey. J Obstetr Gynecol India. 2021;71(1):33-7. 
14. Hoque R. Major mental health problems of undergraduate students in a private university of Dhaka, Bangladesh \%J European Psychiatry. Eur Psychiatry. 2015;30(S1):1.

15. Mondol MS, Mohiuddin MG. Confronting Covid-19 with a paradigm shift in teaching and learning: a study on online classes. Int J Soc Polit Econ Res. 2020;7(2):231-47.

16. Sultana N. Stress and depression among undergraduate medical students of Bangladesh. Bangladesh J Med Educ. 2011;2(1):6-9.

17. Islam MA, Barna SD, Raihan H, Khan MNA, Hossain M. Depression and anxiety among university students during the COVID-19 pandemic in Bangladesh: a web-based cross-sectional survey. PLoS ONE. 2020;15(8):e0238162.

18. Safa F, Anjum A, Hossain S, Trisa TI, Alam SF, Rafi MA, et al. Immediate psychological responses during the initial period of the COVID-19 pandemic among Bangladeshi medical students. Child Youth Serv Rev. 2021;122:105912.

19. Islam MS, Sujan MSH, Tasnim R, Sikder MT, Potenza MN, Van Os J. Psychological responses during the COVID-19 outbreak among university students in Bangladesh. PLoS ONE. 2020;15(12):e0245083.

20. Islam S, Akter R, Sikder T, Griffiths MD, Addiction. Prevalence and factors associated with depression and anxiety among first-year university students in Bangladesh: a cross-sectional study. 2020:1-14.

21. NIPORT. Bangladesh Demography and Health Survey. 2017-2018.

22. Haque M, Das C, Ara R, Alam M, Ullah S, Hossain Z. Prevalence of generalized anxiety disorder and its effect on daily living in the rural community of Rajshahi. J Teach Assoc. 2018;27(1):14-23.

23. Hossain S, Anjum A, Uddin ME, Rahman MA, Hossain MF. Impacts of socio-cultural environment and lifestyle factors on the psychological health of university students in Bangladesh: a longitudinal study. J Affect Disord. 2019;256:393-403.

24. Omani-Samani R, Maroufizadeh S, Ghaheri A, Navid B. Generalized anxiety disorder-7 (GAD-7) in people with infertility: a reliability and validity study. Middle East Fertil Soc J. 2018;23(4):446-9.

25. Eysenbach G. Correction: improving the quality of web surveys: the checklist for reporting results of internet E-Surveys (CHERRIES). J Med Internet Res. 2012;14(1):e8.

26. Sudom K, Guérin E, Lee JE. Gender-related differences in mental health of Canadian Armed Forces members during the COVID-19 pandemic. J Mil Vet Fam Health. 2021;7(S1):46-57.

27. Islam MS, Ferdous MZ, Potenza MN. Panic and generalized anxiety during the COVID-19 pandemic among Bangladeshi people: an online pilot survey early in the outbreak. J Affect Disord. 2020;276:30-7.

28. Blom V, Lönn A, Ekblom B, Kallings LV, Väisänen D, Hemmingsson E, et al. Lifestyle habits and mental health in light of the two COVID-19 pandemic waves in Sweden, 2020. Int J Environ Res Public Health. 2021;18(6):3313.

29. Ravens-Sieberer U, Kaman A, Erhart M, Devine J, Hölling H, Schlack R, et al. Quality of life and mental health in children and adolescents during the first year of the COVID-19 pandemic in Germany: Results of a two-wave nationally representative study. Available at SSRN 3798710. 2021.

30. Chen L, Wang L, Qiu XH, Yang XX, Qiao ZX, Yang YJ, et al. Depression among Chinese university students: prevalence and socio-demographic correlates. PLoS ONE. 2013;8(3):e58379.

31. Sahu KK, Mishra AK, Lal A. Trajectory of the COVID-19 pandemic: chasing a moving target. Ann Transl Med. 2020;8(11):694.

32. Md MI, Md FI, Md SJ. Epidemiological comparison of the first and second wave of COVID-19 Pandemic in Dhaka, Bangladesh: a crosssectional study among suspected cases. J Adv Biotechnol Exp Ther. 2021;5(1):115-25.

33. Li Y, Zhao J, Ma Z, McReynolds LS, Lin D, Chen Z, et al. Mental health among college students during the COVID-19 pandemic in China: a 2-wave longitudinal survey. J Affect Disord. 2021;281:597-604.

34. Lorant V, Smith P, Van den Broeck K, Nicaise P. Psychological distress associated with the COVID-19 pandemic and suppression measures during the first wave in Belgium. BMC Psychiatry. 2021;21(1):1-10.

35. Hoque MN, Hannan A, Imran S, Alam MA, Matubber B, Saha SM. Anxiety and its determinants among undergraduate students during E-learning in Bangladesh Amid Covid-19. J Affect Disord Rep. 2021;6:100241.

36. Ochnik D, Rogowska AM, Kuśnierz C, Jakubiak M, Schütz A, Held MJ, et al. A comparison of depression and anxiety among university students in nine countries during the COVID-19 pandemic. J Clin Med. 2021;10(13):2882.

37. Ahmed O, Ahmed MZ, Alim SM, Khan MA, Jobe MC. COVID-19 outbreak in Bangladesh and associated psychological problems: an online survey. Death Stud. 2020:1-10.

38. Khan AH, Sultana MS, Hossain S, Hasan MT, Ahmed HU, Sikder MT. The impact of COVID-19 pandemic on mental health \& wellbeing among home-quarantined Bangladeshi students: a cross-sectional pilot study. J Affect Disord. 2020;277:121-8.

39. Sayeed A, Kundu S, AI Banna MH, Hasan MT, Begum MR, Khan MS, et al. Mental health outcomes during the COVID-19 and perceptions towards the pandemic: findings from a cross sectional study among Bangladeshi students. Child Youth Serv Rev. 2020;119:105658.

40. Sudha G, Beena ET, Jawahar M, Josephine Arockia Selvi K, Sivasubramaniam S, Weiss MJ. Perceptions of gender and tuberculosis in a south Indian urban community. Indian J Tuberc. 2008;55:9-14.

41. Sultana MS, Khan AH, Hossain S, Ansar A, Sikder MT, Hasan M, et al. Prevalence and predictors of post-traumatic stress symptoms and depressive symptoms among Bangladeshi students during COVID-19 mandatory home confinement: a nationwide cross-sectional survey. Child Youth Serv Rev. 2021;122:105880.

42. Hamadani JD, Hasan MI, Baldi AJ, Hossain SJ, Shiraji S, Bhuiyan MSA, et al. Immediate impact of stay-at-home orders to control COVID-19 transmission on socioeconomic conditions, food insecurity, mental health, and intimate partner violence in Bangladeshi women and their families: an interrupted time series. Lancet Glob Health. 2020;8(11):e1380-9.

43. Malhotra S, Shah R. Women and mental health in India: an overview. Indian J Psychiatry. 2015;57(Suppl 2):S205.

44. Mei S, Yu J, He B, Li J. Psychological investigation of university students in a university in Jilin province. Med Sci. 2011;24(05):84-6.

45. Rabbani F, Khan HA, Piryani S, Khan AR, Abid FJm. Psychological and social impact of COVID-19 in Pakistan: Need for Gender Responsive Policies. medRxiv. 2020.

46. Hou F, Bi F, Jiao R, Luo D, Song K. Gender differences of depression and anxiety among social media users during the COVID-19 outbreak in China: a cross-sectional study. BMC Public Health. 2020;20(1):1-11.

47. Koly KN, Sultana S, Iqbal A, Dunn JA, Ryan G, Chowdhury AB. Prevalence of depression and its correlates among public university students in Bangladesh. J Affect Disord. 2021;282:689-94. 
48. Lasheras I, Gracia-García P, Lipnicki DM, Bueno-Notivol J, López-Antón R, de la Cámara C, et al. Prevalence of anxiety in medical students during the covid-19 pandemic: a rapid systematic review with meta-analysis. Int J Environ Res Public Health. 2020;17(18):6603.

49. Saddik B, Hussein A, Sharif-Askari FS, Kheder W, Temsah M-H, Koutaich RA, et al. Increased levels of anxiety among medical and nonmedical university students during the COVID-19 pandemic in the United Arab Emirates. Risk Manag Healthc Policy. 2020;13:2395.

50. Wathelet M, Duhem S, Vaiva G, Baubet T, Habran E, Veerapa E, et al. Factors associated with mental health disorders among university students in France confined during the COVID-19 pandemic. JAMA Netw Open. 2020;3(10):e2025591.

51. Cao W, Fang Z, Hou G, Han M, Xu X, Dong J, et al. The psychological impact of the COVID-19 epidemic on college students in China. Psychiatry Res. 2020;287:112934.

52. Liu J, Zhu Q, Fan W, Makamure J, Zheng C, Wang JJ. Online mental health survey in a medical college in China during the COVID-19 outbreak. Front Psychiatry. 2020;11:459.

53. Tian-Ci Quek T, Tam W-S, Tran B, Zhang M, Zhang Z, Su-Hui Ho C, et al. The global prevalence of anxiety among medical students: a metaanalysis. Int J Environ Res Public Health. 2019;16(15):2735.

54. Alvi T, Assad F, Ramzan M, Khan FA. Depression, anxiety and their associated factors among medical students. J Coll Physicians Surg Pak. 2010;20(2):122-6.

55. Kashani JH, Priesmeyer M. Differences in depressive symptoms and depression among college students. Am J Psychiatry. 1983;140(8):1081-2.

56. Othieno CJ, Okoth RO, Peltzer K, Pengpid S, Malla LO. Depression among university students in Kenya: prevalence and sociodemographic correlates. J Affect Disord. 2014;165:120-5.

57. Amponsah M, Owolabi H. Perceived stress levels of fresh university students in Ghana: A case study. J Educ Soc Behav Sci. 2011:153-69.

58. Shao GN, Kim H, Imran S. https://www.sciencedirect.com/science/article/abs/pii/S092633731500346X. 2016.

59. Sahu P. Closure of universities due to coronavirus disease 2019 (COVID-19): impact on education and mental health of students and academic staff. Cureus. 2020;12(4):e7541.

60. Hadi A. Women's productive role and marital violence in Bangladesh. J Fam Violence. 2005;20(3):181-9.

61. Ross CE, Mirowsky J. Sex differences in the effect of education on depression: resource multiplication or resource substitution? Soc Sci Med. 2006;63(5):1400-13.

62. Scarinci IC, Beech BM, Naumann W, Kovach KW, Pugh L, Fapohunda B. Depression, socioeconomic status, age, and marital status in black women: a national study. Ethn Dis. 2002;12(3):421-8.

63. Reddy V, Karri SR, Jezreel T, Afeen S, Khairkar P. Psychosocial Impact of COVID-19 lockdown on mental wellbeing among 11 states of India: a Markov modeling approach. J Psychiatry Psychiatr Disord. 2020;4(4):158-74.

Publisher's Note Springer Nature remains neutral with regard to jurisdictional claims in published maps and institutional affiliations. 